\title{
Melanoma-Part 1: epidemiology, risk factors, and prevention
}

\author{
Veronique Bataille, ${ }^{1,2}$ Esther de Vries ${ }^{3,4}$
}

${ }^{1}$ West Hertfordshire Hospitals NHS Trust, Hemel Hempstead HP2 4AD

${ }^{2}$ Twin Research and Genetic Epidemiology Unit, King's College London SE1 7EH

${ }^{3}$ Department of Public Health, Erasmus MC University, Medical Center, Rotterdam, Netherlands

${ }^{4}$ Department of Dermatology,

Erasmus MC University Medical

Center, Rotterdam

Correspondence to: V Bataille

bataille@doctors.org.uk

Cite this as: BMJ 2008;337:a2249 doi:10.1136/bmj.a2249
The incidence of melanoma has risen over the past 30 years in most white populations. ${ }^{12}$ However, in some parts of the world incidence rates are stable or falling. Although large scale primary prevention programmes such as public health education campaigns aimed at reducing exposure to sun may lead to reduced incidence, such programmes have not yet been proved effective. However, melanoma is highly amenable to secondary prevention through early detection. Being able to recognise a melanoma early is an important skill for primary healthcare practitioners. In this first article of a two part series on melanoma, we examine the evidence on the genetic epidemiology of melanoma and outline the complex interactions between incidence, mortality, and survival trends; we also discuss the evidence for prevention.

\section{How common is melanoma?}

Although melanoma remains a relatively rare tumour, many European countries show an annual increase in incidence above $2 \%$; in some northern European countries, however, no significant increase has been observed. ${ }^{1}$ Between 1992 and 2005, the annual increase in incidence rates of melanoma in the United States was $2.3 \%$ for all races and $2.8 \%$ in white populations. ${ }^{3}$ Queensland in Australia had an increase in incidence of $1.4 \%$ for males and $0.7 \%$ for females during 1982 to $2001 .{ }^{4}$ Most recent incidence data show that in some parts of the US, Australia, and the United Kingdom, incidence of melanoma has reached a plateau and even started to decrease. ${ }^{35}$

\section{Should we be concerned by a rising incidence of melanoma?}

Examining incidence, mortality, and survival trends for melanoma together can produce a clear picture of the scale of the problem. Incidence has been inflated by early lesions that have little potential to spread and have a low impact on mortality. ${ }^{6}$ In countries with health education campaigns related to skin cancer, much of the rise in incidence can be attributed to very thin melanoma lesions or melanomas in situ.

In Australia, for example, an epidemiological study that reviewed cancer registries showed that the rate of increase of melanoma in situ has been about $9 \%$ a year compared with only $2.8 \%$ for invasive melanomas. ${ }^{2}$ Over the same period the incidence of thin melanomas has increased faster than that of thick tumours, which have not become much more common. ${ }^{2}$ Although thin lesions are diagnosed on average five to 10 years earlier than thick ones, this does not necessarily suggest that they are detected shortly before they become invasive. ${ }^{2}$ Earlier diagnosis is more likely to reflect a trend in younger patients attending screening.

In contrast with the data on rising incidence, mortality curves for melanoma have been fairly flat over the past 30 years in most white populations and have even begun to fall in the US, Australia, Scandinavia, and the UK. ${ }^{17}$ Early melanomas (often in situ tumours) are increasingly being excised, and comparisons of incidence and mortality data suggest that many borderline lesions may never progress if left alone. $^{2}$

\section{Who gets melanoma?}

The mean age of presentation with melanoma is about 55 years, but this may vary according to thickness and histological subtype. ${ }^{3}$ Superficial spreading melanomas, usually thinner tumours, are seen in younger individuals, whereas nodular melanoma and lentigo maligna melanoma tend to affect older individuals.

Men and women are generally equally likely to be diagnosed with melanoma, but in some countries a

\section{QUESTIONS FOR FUTURE RESEARCH}

- Why does body site and survival differ so much by sex in melanoma?

- Will the discovery of low penetrance susceptibility genes for melanoma help us in targeting populations at risk?

- Is a reduction in people's exposure to sun reducing the incidence of melanoma?

- Are recent trends in melanoma explained by increased surveillance and ascertainment of very early noninvasive tumours and not by a genuine increase in the incidence? 


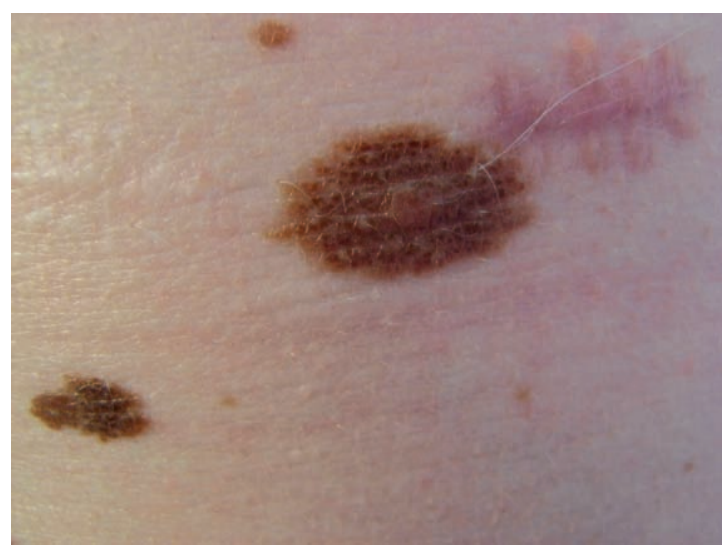

Fig 1 Atypical naevus with diameter of more than $5 \mathrm{~mm}$ and hazy border

slight female preponderance is seen. ${ }^{1}$ The most common site for women is the legs and for men the trunk..$^{89}$ That the difference is attributable to different patterns of sun exposure in men and women is unlikely because a difference in site according to sex is seen in countries with differing sun exposure and clothing habits. For example, the site distribution of melanoma according to sex is similar in Australia, the UK, and Israel despite different skin exposure habits between the three countries. ${ }^{10}$ Site differences according to sex are likely to reflect genetic differences as naevi are also differently distributed between boys and girls from an early age. ${ }^{11}$

\section{What are the risk factors for melanoma? Exposure to sun}

Although sun exposure has long been suspected to be a risk factor for melanoma, the relation between sun exposure and melanoma is complex. The evidence that sun exposure may be associated with melanoma is apparent when comparing incidence in Australia and sunny parts of the US with Europe. ${ }^{3}$ The incidence of melanoma is three to four times higher in Queensland, Australia, than in the $\mathrm{UK}^{2}{ }^{2}$ However, a significant association between sun exposure behaviour and

\section{Box 1 What to look for when a naevus changes}

\section{Change in size}

Naevi may change in size over years, but any change over weeks or months is suspicious

\section{Change in colour}

Melanomas often show irregular pigment in a lesion, with shades of black, brown, grey, and pink. In nodular melanoma the lesion is often black throughout. Rarely a melanoma can present as a non-pigmented red nodule (amelanocytic melanoma); this is more likely on hands and feet

\section{Change in outline}

Melanomas often show a geographical outline with a sharp cut-off from normal skin Itching

Itching may be a late sign and is often unreliable as many benign naevi intermittently itch Bleeding

Bleeding is also a late sign and is often present in advanced melanoma melanoma is less easy to show in a case-control setting. ${ }^{12} 13$ A meta-analysis of melanoma case-control studies found low relative risks associated with various measures of exposure to ultraviolet radiation, and the relation with sunshine was not dose dependent. ${ }^{14}$ Sharp, short bursts of acute exposure in childhood, and severe sunburn, were most strongly associated with melanoma. However, cumulative occupational exposure seemed to be protective in some white populations. Odds ratios associated with sun exposure are often no longer significant after adjustment for skin type, which supports a hypothesis that host response to ultraviolet radiation is more important than dose of sun exposure.

\section{Use of sun beds}

Melanoma is controversially associated with use of sunbeds. Some epidemiological studies have not found sunbed use to be a significant risk, whereas others have found small increased risks. ${ }^{1315-17}$

Sunbed use seems to be most detrimental before the age of 20, and current recommendations are that sunbeds should be avoided, especially in relating to premature skin ageing. ${ }^{17}$ Individuals with red hair and freckles, or multiple atypical naevi, with or without a family history of melanoma, should avoid sunbeds since their risks of developing both melanoma and nonmelanoma skin cancer are already significantly increased. ${ }^{18}$

\section{Skin pigmentation}

Having fair skin with a poor ability to tan, or a freckled complexion with or without red hair, doubles a person's risk of melanoma. ${ }^{18}$ Polymorphisms in the melanocortin 1 receptor (MC1R) gene have been linked to fair skin and red hair and have been associated with an increased risk of melanoma. ${ }^{1920}$

Melanomas present rarely in non-white people, in whom the incidence is 10 to 20 times lower than in white people. ${ }^{3}$ Body site and histological subtypes differ between white and non-white people. The latter more frequently present with lentiginous and nodular

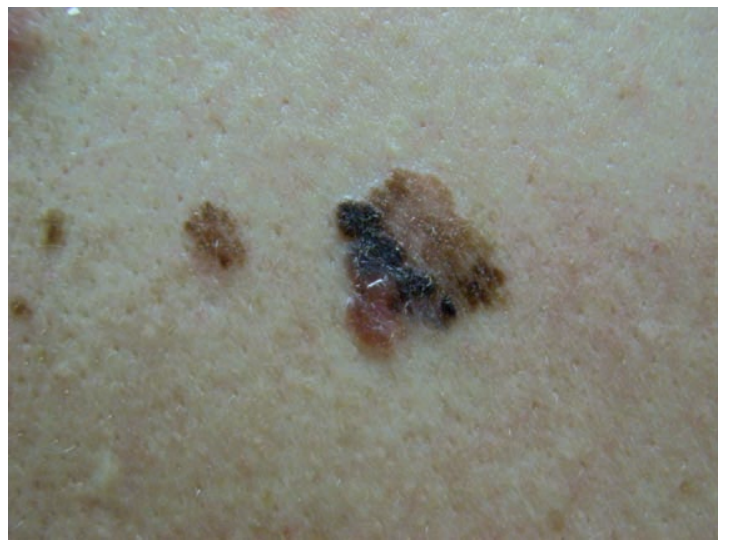

Fig 2 | Superficial spreading melanoma with a well demarcated edge and irregular pigmentation 
melanoma on the palms and soles and rarely on other parts of the body. ${ }^{321}$

\section{Naevi}

Naevi are the most powerful predictor of risk of melanoma. A meta-analysis of observational studies found that an individual who has more than 100 common naevi or more than two atypical naevi has a fivefold to 20-fold increased risk of melanoma. ${ }^{22}$ Unlike melanoma, atypical naevi (fig 1) are usually symmetrical and do not have a sharp edge with geographical border; asymmetry and sharp edged borders are clear signs of malignant transformation (fig 2) ${ }^{23}$ Furthermore, naevi remain static whereas melanoma change in size, shape, or colour over weeks or months.

In younger individuals atypical naevi on the trunk and limbs are more predictive of superficial melanoma than of nodular melanoma or lentigo maligna melanoma, which affect older individuals with more sun damage and fewer naevi. ${ }^{24 \mathrm{w} 1}$ The presence of multiple common naevi and atypical naevi denotes the phenotype for atypical mole syndrome, which may indicate a genetic susceptibility to melanoma (boxes 1 and 2). This phenotype is found in about $2 \%$ of a healthy population in the UK. ${ }^{\text {2 }}$

Two twin studies suggest that naevi are under considerable genetic control, with a $60 \%$ concordance in numbers of naevi in identical twins. ${ }^{\text {w3 w4 }}$ A cross sectional twin study of over 2000 individual twins showed that naevi undergo senescence with age, with a decrease in numbers from the age of 30 to 40 years, but that in patients with the atypical mole syndrome the number of naevi can remain high after middle age. ${ }^{\text {w3 }}$ In patients with the syndrome, naevi are also often found on unusual sites, such as scalp, buttocks, feet, hands, and irises. ${ }^{\mathrm{w} 5}$

\section{Solar keratoses}

Solar keratoses carry a relative risk for melanoma of 2 to $4 .^{\mathrm{w} 6 \mathrm{w} 7}$ The presence of naevi and solar keratoses is often mutually exclusive, suggesting different pathways to melanoma-via an excess of naevi in younger individuals or via chronic sun damage and cumulative sun exposure in older subjects. ${ }^{24 \mathrm{w} 6 \mathrm{w} 7}$

\section{Box 2 Phenotype for atypical mole syndrome}

\section{What are the features of the phenotype?}

- More than 100 common naevi ( $\leq 2 \mathrm{~mm}$ in diameter)

- More than two atypical naevi ( $\geq 5 \mathrm{~mm}$ in diameter)

- Naevi on unusual sites such as breasts in females, buttocks, scalp, ears, dorsum of feet

\section{When the phenotype is present}

- If there is also a family history of melanoma or multiple cancers, referral to a dermatologist is recommended

- Removal of atypical naevi just to confirm histological dysplasia is not helpful. Lesions should be removed only if early melanoma is suspected and only by an experienced clinician

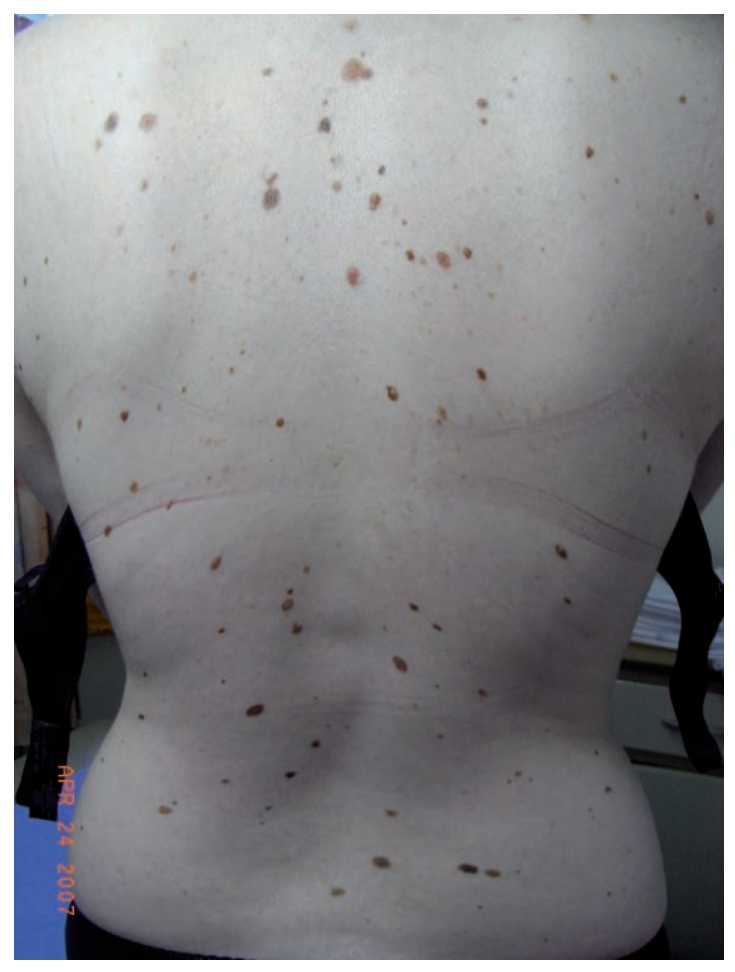

Fig 3 Atypical mole syndrome with many atypical naevi on trunk

\section{Family history of melanoma and genetic factors}

Five to ten per cent of individuals with melanoma have a family history of melanoma. ${ }^{\text {w5 }}$ In families with melanoma, affected individuals often have the atypical mole syndrome phenotype (fig 3). About a quarter of all families with melanoma have been linked to mutations in the tumour suppressor gene CDKN2A/ p16 on chromosome 9p21, which affects the germline and is therefore transmitted through generations. ${ }^{\text {w8 }}$ Mutation screening outside the research setting is not recommended as the prevalence of germline CDKN2A mutations is very low and does not alter management. ${ }^{23}$ In some families with melanoma, susceptibility to some other cancers - such as pancreas, brain, and breast cancer-is increased, so taking a thorough family history of cancers in patients with melanoma is important. ${ }^{\text {} 9}$

\section{Hormonal factors}

Mortality studies have shown that women have thinner tumours and survive melanoma better than men even after adjustment for Breslow thickness, ulceration, and body site, which suggests that there may be $\mathrm{X}$ linked variable gene expression or hormonal factors affecting melanoma survival. ${ }^{9 \mathrm{w} 10}$ Anecdote reports melanoma behaving aggressively during pregnancy and metastases manifesting themselves during pregnancy. However, a metaanalysis that examined data on over 5000 female participants showed that risk of melanoma was not affected by pregnancy, oral contraception, or hormone replacement. ${ }^{\text {11 }}$ 


\section{Can melanoma be prevented?}

Primary prevention-benefits and harms

Health education campaigns aimed at reducing people's exposure to sun began in Australia in the 1980s and were soon adopted elsewhere in the world. There is, as yet, no direct evidence that reducing sun exposure has had an effect on melanoma incidence. Some commentators have suggested that the slowing down and even downturn in incidence in melanoma in some parts of the world may be due to the health education programmes, but the downturn has been seen in parts of the world where levels of sun exposure and public health campaigns vary. ${ }^{4}$ Furthermore, the lag time between change in behaviour and the recent downturns in incidence may have been too short for health education to be the sole explanation.

A recent $B M J$ editorial raised a concern that drastic reduction of sun exposure in temperate climates may have an impact on health because of low vitamin $\mathrm{D}$ levels. ${ }^{\text {w12 }}$ Vitamin D is important for bone metabolism but has also been shown to have protective effects against inflammation, cancer, psychiatric diseases, and autoimmune disease. Avoiding sunburn and excessive sun exposure without protection seems to be the most important message in skin cancer prevention without

\section{ADDITIONAL EDUCATIONAL RESOURCES}

\section{Resources for patients}

- GenoMEL (www.genomel.org/english/patientInformation.htm)—GenoMEL (Melanoma Genetics Consortium) has available a booklet, Melanoma: Dealing with the Diagnosis

- Royal College of Physicians. Prevention, diagnosis, referral and management of melanoma of the skin. Concise guideline No 7. www.rcplondon.ac.uk/pubs/brochure. aspx?e=216

- British Association of Dermatologists (www.bad.org.uk/public/leaflets/melanoma. asp)—Leaflet and contacts for support

- Cancer Research UK (www.cancerhelp.org.uk/help/default.asp?page=2788)—Advice on melanoma

Resources for health professionals

- GenoMEL (www.genomel.org/english/physicianInformation.htm)—GenoMEL (Melanoma Genetics Consortium) gives information and references on melanoma

- National Cancer Institute. Surveillance epidemiology and end results. (http://seer. cancer.gov/faststats/selections.php? run\&output $=2 \&$ data $=1 \&$ statistic $=2 \&$ year $=200802 \&$ race $=2 \&$ sex $=1 \&$ age $=1 \&$ series $=$ $=1 \&$ statistic $=2 \&$ year $=200802 \&$ race $=2 \&$ sex $=1 \&$ age $=1 \&$ series=cancer \&cancer=53)

- Cancer Research UK (http://info.cancerresearchuk.org/cancerstats/types/skin/ incidence/)-Gives statistics on skin cancer

- Information Services Division Scotland (www.isdscotland.org/isd/1048. html\#Malignant\%20melanoma\%20of\%20the\%20skin)—Data, reports, and factsheets.

- Melanoma Molecular Map Project (www.mmmp.org)—Six interconnected databanks for the interactive collection, update, and consultation of the translational and clinical information on melanoma biology and treatment

- National Institute for Health and Clinical Excellence. Skin tumours including melanoma. (Cancer service guidance.) 2006. www.nice.org.uk/Guidance/CSGSTIM

- Department of Health. Guidelines for the appointment of general practitioners with special interests in the delivery of clinical services: dermatology. 2003. www.dh.gov.uk/ en/Publicationsandstatistics/Publications/PublicationsPolicyAndGuidance/ DH_4008233

\section{SUMMARY POINTS}

The incidence of melanoma is rising, but most of the rise is caused by very thin melanomas

The most powerful risk factor for melanoma is the number of naevi

There are two phenotypic pathways to melanoma: via naevi or via sun damage

Melanoma mortality has been relatively stable over the past 30 years

A reduction in people's exposure to sun has not led to a significant reduction in the incidence of melanoma, and sun avoidance may be detrimental; secondary prevention with early detection of melanoma saves lives

advocating keeping away from the sun altogether. ${ }^{\mathrm{wl}}$ More research is needed in this field to understand not only the relation between melanoma and sun exposure but also the potential detrimental effects of vitamin D deficiency after sun avoidance.

Secondary prevention-early identification and treatment Melanoma is amenable to secondary prevention as tumours are highly visible with a good prognosis if detected early. Secondary prevention is a more efficient way to reduce mortality from melanoma than primary prevention as the latter has not yet been proved to reduce incidence or mortality significantly. However, for secondary prevention to be successful, educating the public and health professionals to recognise melanoma early is crucial.

\section{Is there any benefit in screening?}

Screening strategies have not yet been formally assessed as the design of such trials is difficult. Individuals with multiple atypical naevi, a family history of melanoma, and/or multiple cancers should be referred to a dermatologist for screening as their risk of melanoma is significantly increased. ${ }^{23}$ However, melanoma is an uncommon cancer and population screening may not yield a substantial number of cases. Public health campaigns depicting melanoma as a serious killer are no longer accurate as more than $80 \%$ of patients with melanoma now survive their disease, with most melanomas being treated with relatively minor surgery alone. ${ }^{17}$ Concern has been expressed that aggressive public health campaigns lead to anxiety related increases in the excision of benign lesions and melanoma in situ. ${ }^{\mathrm{w} 14}$ In Australia, where melanoma screening is most active, a sharp rise in melanoma in situ and very thin melanomas has been observed over the past 10 years, thus inflating incidence figures. ${ }^{2}$ There is certainly a need to educate the public on what to look for when a mole changes, while avoiding generating anxiety and the subsequent removal of too many benign lesions.

Contributors: VB and EdeV both contributed to the writing and editing o the article and approved the final version. The guarantor is VB. Competing interests: None declared. 
Provenance and peer review: Commissioned; externally peer reviewed. Patient consent obtained.

1 Karim-Kos HE, de Vries E, Soerjomataram I, Lemmens V, Siesling S, Coebergh JW. Recent trends of cancer in Europe: a combined approach of incidence, survival and mortality for 17 cancer sites since the 1990s. Eur J Cancer 2008;44:1345-89.

2 Coory M, Baade P,Aitken J, Smithers M, McLeod GR, Ring I. Trends in situ and invasive melanoma in Queensland, Australia 1982-2002. Cancer Causes Control 2006;17:21-2.

3 National Cancer Institute. Surveillance epidemiology and end results. Fast stats. http://seer.cancer.gov/faststats/selections.php? run\&output $=2 \&$ data $=1$ \&statistic $=2$ \&year $=200802$ \&ace $=2 \&$ sex $=1 \&$ $=2 \&$ data $=1 \&$ statistic $=2 \&$ year $=200802 \&$ race $=2 \&$ sex $=1 \&$ age $=1 \&$ ser $=1 \&$ statistic $=2 \&$ year $=200802 \&$ race $=2 \&$ sex $=1 \&$ age $=1 \&$ series $=$ can $=2 \&$ year $=200802 \&$ race $=2 \&$ sex $=1 \&$ age $=1 \&$ series $=$ cancer $\&$ can cer $=53$

4 Whiteman DC, Bray CA, Siskind V, Green AC, Hole DJ, Mackie RM. Changes in the incidence of cutaneous melanoma in the west of Scotland and Queensland, Australia: hope for health promotion. Eur J Cancer Prev 2008;17:243-50.

5 Australian Institute of Health and Welfare. Australian cancer incidence and and mortality workbooks. ACIM Books Version 1.2007. www.aihw.gov.au/cancer/data/acim_books/melanoma_of_skin. xls.

6 Melanoma Molecular Map Project. TNM staging. www.mmmp.org/ MMMP/import.mmmp?page=tnm_staging.mmmp.

7 Giles G, Armstrong BK, Burton RC, Staples MR, Thursfield VJ. Has mortality from melanoma stopped rising in Australia? Analysis of trends between 1931 and 1994. BMJ 1996;312:1121-5.

8 Cancer Research UK. Melanoma skin cancer. www.cancerhelp.org. uk/help/default.asp?page $=2788$.

9 De Vries E, Nijsten E, Visser O, Bastiaanet E, van Hatten S, Janssen-Heijnen ML, et al. Superior survival of females amongst 10,358 Dutch melanoma patients is independent of Breslow thickness, histological type and tumour site. Ann Oncol 2008;19:583-9.

10 Bulliard JL, Cox B, Elwood JM. Comparison of the site of melanoma in New Zealand and Canada. Int J Cancer 1997;72:231-5.

11 Harrison S, Mackie R-M, MacLennan R. Development of melanocytic nevi in the first three years of life. J Natl Cancer Inst 2000;92:1436-8.
12 Bataille V, Winnett A, Sasieni P, Newton Bishop JA, Cuzick J. Exposure to the sun and sunbeds and the risk of cutaneous melanoma in the UK: a case-control study. Eur J Cancer 2004;40:429-35.

13 Holly EA, Aston DA, Cress RD, Ahn DK, Kristiansen JJ. Cutaneous melanoma in women. I. Exposure to sunlight, ability to tan, and other risk factors related to ultraviolet light. Am J Epidemiol 1995;141:923-33.

14 Gandini S, Sera F, Cattaruzza MS, Pasquini P, Picconi O, Boyle P, et al. Metaanalysis of risk factors for cutaneous melanoma: II. Sun exposure. Eur J Cancer 2005;41:45-60.

15 Westerdahl J, Olsson H, Måsbäck A, Ingvar C, Jonsson N, Brandt L, et al. Use of sunbeds or sunlamps and malignant melanoma in southern Sweden. Am J Epidemiol 1994;140:691-9.

16 Bataille V, Boniol M, de Vries E, Severi G, Brandberg Y, Sasieni P, et al. A multicentre epidemiological study on sunbed use and cutaneous melanoma in Europe. EurJ Cancer 2005;41:2141-9.

17 Autier P. Cutaneous malignant melanoma. Facts about sunbeds and sunscreens. Expert Rev Anticancer Ther 2005;5:881-33.

18 Bliss JM, Ford D, Swerdlow AJ, Armstrong BK, Cristofolini M, Elwood JM, et al. Risk of cutaneous melanoma associated with pigmentation characteristics and freckling: systematic overview of 10 case-control studies. International Melanoma Analysis Group (IMAGE). Int J Cancer 1995;62:367-76.

19 Palmer JS, Duffy DL, Box NF, Aitken JF, O'Gorman LE, Green AC, et al. Melanocortin-1-receptor polymorphisms and risk of melanoma: is the association explained solely by pigmentation phenotype? Am J Hum Genet 2000;66:176-86.

20 Mössner R, Anders N, König IR, Kruger U, Schmidt D, Berking C, et al. Variations of the melanocortin-1 receptor and the glutathione-S transferase T1 and M1 genes in cutaneous malignant melanoma. Arch Dermatol Res 2007;298:371-9.

21 Cress RD, Holly EA. Incidence of cutaneous melanoma among nonHispanic whites, Hispanics, Asians and blacks. An analysis of California Cancer Registry data, 1988-1993. Cancer Causes Control 1997;8:246-52

22 Gandini S, Sera F, Cattaruzza MS, Pasquini P, Albeni D, Boyle P, et al. Meta-analysis of risk factors for cutaneous melanoma: I. Common and atypical naevi. Eur J Cancer 2005b;41:28-4.

23 Newton Bishop JA, Gruis NA. Genetics: what advice for patients who present with a family history of melanoma? Semin Oncol 2007;34:452-9.

24 Cho E, Rosner BA, Colditz GA. Risk factors for melanoma by body site. Cancer Epidemiol Biomarkers Prev 2005;14:1241-4.

\section{The peril of treating your progeny}

Lucy was in her 30 s with a promising career as an internet programmer and a lucrative offer to work in Asia. She also zealously volunteered her time and service to an international health organisation. She was the daughter of a practising doctor, who was successful in his own right.

However, a recent history of headaches and photophobia had increased in intensity to the point of Lucy being hospitalised. The results of imaging of the brain, performed at a hospital where her father worked, were reported as within normal limits, and analysis of her cerebrospinal fluid revealed a few mononuclear cells. She was subsequently discharged with analgesics and a diagnosis of aseptic meningitis.

Her symptoms progressed, however, and she became virtually blind. An ophthalmologist diagnosed progressive outer retinal necrosis from herpes zoster infection and immediately ordered an HIV serology test, which produced a positive result. This also showed a high virologic load and a very low $\mathrm{CD} 4$ cell count indicative of advanced disease. Her cerebrospinal fluid obtained at her previous hospital admission showed the presence of varicella zoster virus. Her retinal necrosis was extensive, and - despite highly active antiretroviral therapy, intraocular and systemic foscarnet and ganciclovir, and a silicon tamponade to stabilise her retina - she ultimately became completely blind.
Lucy for many years had had a history of sinopulmonary infections and an episode of multidermatomal shingles, and these infections were either observed or treated presumptively with antibiotics by her father or his associates. Lucy is now considering her vocational goals as she had to relinquish her career as an internet programmer and is adjusting to the emotional trauma and physical limitations of her blindness.

It is easy to be judgmental in hindsight, but her father, filled with regret and guilt, struggled to hold back the tears as he said to me, "My duty was to be her father, not her doctor."

I am now the father of two small children, and those words of Lucy's father echo strongly in my own philosophy towards their health. My emotional connection with my children will always preclude an objective and optimal assessment. They have their own family practitioner; not one who is a close friend of mine but a doctor who is highly recommended by my neighbours.

Suran Fernando clinical immunologist, Royal North Shore Hospital, St Leonards, Australia suran@tpg.com.au

Patient consent obtained.

Cite this as: BMJ 2008;337:a2307 